\title{
Constructing new optimal entanglement witnesses
}

\author{
Dariusz Chruściński, Justyna Pytel and Gniewomir Sarbicki \\ Institute of Physics, Nicolaus Copernicus University, \\ Grudziadzka 5/7, 87-100 Toruń, Poland
}

\begin{abstract}
We provide a new class of indecomposable entanglement witnesses. In $4 \times 4$ case it reproduces the well know Breuer-Hall witness. We prove that these new witnesses are optimal and atomic, i.e. they are able to detect the "weakest" quantum entanglement encoded into states with positive partial transposition (PPT). Equivalently, we provide a new construction of indecomposable atomic maps in the algebra of $2 k \times 2 k$ complex matrices. It is shown that their structural physical approximations give rise to entanglement breaking channels. This result supports recent conjecture by Korbicz et. al.

PACS numbers: 03.65.Ud, 03.67.-a
\end{abstract}

\section{INTRODUCTION}

The interest on quantum entanglement has dramatically increased during the last two decades due to the emerging field of quantum information theory [1]. It turns out that quantum entanglement may be used as basic resources in quantum information processing and communication. The prominent examples are quantum cryptography, quantum teleportation, quantum error correction codes and quantum computation.

Since the quantum entanglement is the basic resource for the new quantum information technologies it is therefore clear that there is a considerable interest in efficient theoretical and experimental methods of entanglement detection (see [2] for the review).

The most general approach to characterize quantum entanglement uses a notion of an entanglement witness (EW) 3, 4]. A Hermitian operator $W$ defined on a tensor product $\mathcal{H}=\mathcal{H}_{A} \otimes \mathcal{H}_{B}$ is called an EW iff 1) $\operatorname{Tr}\left(W \sigma_{\text {sep }}\right) \geq 0$ for all separable states $\sigma_{\text {sep }}$, and 2) there exists an entangled state $\rho$ such that $\operatorname{Tr}(W \rho)<0$ (one says that $\rho$ is detected by $W$ ). It turns out that a state is entangled if and only if it is detected by some EW [3]. There was a considerable effort in constructing and analyzing the structure of EWs [5, 6, 7, 8, 9, 10, 11, 12, 13, [14, 15]. In fact, entanglement witnesses have been measured in several experiments [16, 17]. Moreover, several procedures for optimizing EWs for arbitrary states were proposed [6, 18, 19, 20]. It should be stressed that there is no universal $W$, i.e. there is no entanglement witness which detects all entangled states. Each entangled state $\rho$ may be detected by a specific choice of $W$. It is clear that each EW provides a new separability test and it may be interpreted as a new type of Bell inequality [21]. There is, however, no general procedure for constructing EWs.

Due to the Choi-Jamiołkowski isomorphism [22, 23] any EW corresponds to a linear positive map $\Lambda$ : $\mathcal{B}\left(\mathcal{H}_{A}\right) \rightarrow \mathcal{B}\left(\mathcal{H}_{B}\right)$, where by $\mathcal{B}(\mathcal{H})$ we denote the space of bounded operators on the Hilbert space $\mathcal{H}$. Recall that a linear map $\Lambda$ is said to be positive if it sends a positive operator on $\mathcal{H}_{A}$ into a positive operator on $\mathcal{H}_{B}$. It turns out [3] that a state $\rho$ in $\mathcal{H}_{A} \otimes \mathcal{H}_{B}$ is separable iff $\left(\mathbb{1}_{A} \otimes \Lambda\right) \rho$ is positive definite for all positive maps $\Lambda: \mathcal{B}\left(\mathcal{H}_{B}\right) \rightarrow \mathcal{B}\left(\mathcal{H}_{A}\right)$ (actually this result is based on [24]). Unfortunately, in spite of the considerable effort, the structure of positive maps is rather poorly understood $24,25,26,27,28,29,30,31,32$, 33, 34, 35, 36, 37, 38, 39, 40, 41].

In the present paper we provide a construction of a new class of positive maps in $\mathcal{B}\left(\mathbb{C}^{2 k}\right)$ with $k \geq 2$. Our construction uses the well-known reduction map as a building block. It turns out that for $k=2$ our construction reproduces Breuer-Hall maps [36, 37] but for $k>2$ it gives completely new family of maps. It is shown that proposed maps are indecomposable (i.e. they are able to detect entangled PPT states) and atomic (i.e. they are able to detect "weakly" entangled PPT states). As a byproduct we construct new families of PPT entangled states detected by our maps.

The paper is organized as follows: for pedagogical reason we collect basic definitions and introduce the most important properties of positive maps and entanglement witnesses in Section II. Section III provides basic construction. Then in Section IV we study basic properties of our maps/witnesses (indecomposability, atomicity, optimality). Section $\mathrm{V}$ discusses structural physical approximation (SPA) [42, 43, 44] of our maps. It is shown that the corresponding SPA gives rise to entanglement breaking channels and hence it supports recent conjecture by Korbicz et. al. [44]. Final conclusions are collected in the last Section. 


\section{POSITIVE MAPS, ENTANGLEMENT WITNESSES AND ALL THAT}

For the reader convenience we recall basic definitions and properties which are important throughout this paper.

\section{A. Positive maps}

Let $\Lambda: \mathcal{B}\left(\mathcal{H}_{A}\right) \rightarrow \mathcal{B}\left(\mathcal{H}_{B}\right)$ be a positive linear map. In what follows we shall consider only finite dimensional Hilbert spaces such that $\operatorname{dim} \mathcal{H}_{A}=d_{A}$ and $\operatorname{dim} \mathcal{H}_{B}=d_{B}$. One calls $\Lambda k$-positive if

$$
\mathbb{1}_{k} \otimes \Lambda: M_{k} \otimes \mathcal{B}\left(\mathcal{H}_{A}\right) \longrightarrow M_{k} \otimes \mathcal{B}\left(\mathcal{H}_{B}\right)
$$

is positive. In the above formula $M_{k}$ denotes a linear space of $k \times k$ complex matrices and $\mathbb{1}_{k}: M_{k} \rightarrow M_{k}$ is an identity map, i.e. $\mathbb{1}_{k}(A)=A$ for each $A \in M_{k}$. A positive map which is $k$-positive for each $k$ is called completely positive (CP). Actually, if $d_{A}, d_{B}<\infty$ one shows [22] that $\Lambda$ is $\mathrm{CP}$ iff it is $d$-positive with $d=$ $\min \left\{d_{A}, d_{B}\right\}$.

Definition 1 A positive map $\Lambda$ is decomposable if

$$
\Lambda=\Lambda_{1}+\Lambda_{2} \circ T
$$

where $\Lambda_{1}$ and $\Lambda_{2}$ are $C P$ and $T$ denotes transposition in a given basis. Maps which are not decomposable are called indecomposable (or nondecomposable).

Definition 2 A positive map $\Lambda$ is atomic if it cannot be represented as

$$
\Lambda=\Lambda_{1}+\Lambda_{2} \circ T
$$

where $\Lambda_{1}$ and $\Lambda_{2}$ are 2-positive.

Definition 3 A positive map $\Lambda$ is optimal if and only if for any $C P$ map $\Phi$, the map $\Lambda-\Phi$ is no longer positive.

\section{B. Entanglement witnesses}

Using Choi-Jamiołkowski isomorphism [22, 23] each positive map $\Lambda$ gives rise to entanglement witness $W$

$$
W=d_{A}\left(\mathbb{1}_{A} \otimes \Lambda\right) P_{A}^{+},
$$

where $P_{A}^{+}$denotes maximally entangled state in $\mathbb{C}^{d_{A}} \otimes \mathbb{C}^{d_{A}}$ and $\mathbb{1}_{A}$ denotes an identity map acting on $\mathcal{B}\left(\mathcal{H}_{A}\right)$. One has an obvious
Definition 4 An entanglement witness $W$ defined by (4) is decomposable/indecomposable (atomic) [optimal] $\{k-E W\}$ if and only if the corresponding positive map $\Lambda$ is decomposable/indecomposable (atomic) [optimal] $\{k$-positive $\}$.

It is clear that $W \in \mathcal{B}\left(\mathcal{H}_{A} \otimes \mathcal{H}_{B}\right)$ is a decomposable EW iff

$$
W=A+B^{\Gamma},
$$

where $A, B \geq 0$ and $B^{\Gamma}=\left(\mathbb{1}_{A} \otimes T\right) B$ denotes partial transposition. Witnesses which cannot be represented as in (5) are indecomposable

Let $\psi$ be a normalize vector in $\mathcal{H}_{A} \otimes \mathcal{H}_{B}$. Denote by $\mathrm{SR}(\psi)$ the number of nonvanishig Schmidt coefficients of $\psi$. One has

$$
1 \leq \mathrm{SR}(\psi) \leq d
$$

Now, $W$ is $k-\mathrm{EW}$ iff

$$
\langle\psi|W| \psi\rangle \geq 0
$$

for each $\psi$ such that $\operatorname{SR}(\psi) \leq k$. Evidently, $W \geq 0$ iff $W$ is $d-\mathrm{EW}$. Now, $W$ is atomic if it cannot be represented as

$$
W=W_{1}+W_{2}^{\Gamma},
$$

where $W_{1}$ and $W_{2}$ are 2-EWs. Finally, $W$ is optimal EW iff for any $P \geq 0, W-P$ is no longer EW. Following [6] one has the following criterion for the optimality of $W$ : if the set of product vectors $\psi \otimes \phi \in \mathcal{H}_{A} \otimes \mathcal{H}_{B}$ satisfying

$$
\langle\psi \otimes \phi|W| \psi \otimes \phi\rangle=0,
$$

span the total Hilbert space $\mathcal{H}_{A} \otimes \mathcal{H}_{B}$, then $W$ is optimal.

\section{Detecting quantum entanglement}

Positive maps and EWs are basic tools in detecting quantum entanglement. A state $\rho$ in $\mathcal{H}_{A} \otimes \mathcal{H}_{B}$ is separable if and only if for all positive maps $\Lambda: \mathcal{B}\left(\mathcal{H}_{B}\right) \rightarrow$ $\mathcal{B}\left(\mathcal{H}_{A}\right)$ one has

$$
\left(\mathbb{1}_{A} \otimes \Lambda\right) \rho \geq 0 .
$$

Equivalently, iff for each entanglement witness $W$

$$
\operatorname{Tr}(\rho W) \geq 0 .
$$

Note that entangled PPT states can be detected by indecomposable maps/witnesses only. Let $\sigma$ be a density operator in $\mathcal{H}_{A} \otimes \mathcal{H}_{B}$. Following [45] one introduces its Schmidt number

$$
\operatorname{SN}(\sigma)=\min _{p_{k}, \psi_{k}}\left\{\max _{k} \operatorname{SR}\left(\psi_{k}\right)\right\},
$$


where the minimum is taken over all possible pure states decompositions

$$
\sigma=\sum_{k} p_{k}\left|\psi_{k}\right\rangle\left\langle\psi_{k}\right|
$$

with $p_{k} \geq 0, \sum_{k} p_{k}=1$ and $\psi_{k}$ are normalized vectors in $\mathcal{H}_{A} \otimes \mathcal{H}_{B}$. Note, that if $\sigma=|\psi\rangle\langle\psi|$, then $\operatorname{SN}(\sigma)=\operatorname{SR}(\psi)$. Again one has $1 \leq \operatorname{SN}(\sigma) \leq d$. Suppose now that $\sigma$ is PPT but entangled. Intuitively, the "weakest" quantum entangled encoded in $\sigma$ corresponds to the situation when $\operatorname{SR}(\sigma)=\operatorname{SR}\left(\sigma^{\Gamma}\right)=2$. Such "weakly" entangled PPT states can be detected by atomic maps/witnesses only.

\section{REDUCTION MAP AS A BUILDING BLOCK}

Let us start with an elementary positive map in $\mathcal{B}\left(\mathbb{C}^{n}\right)$ called reduction map [46]

$$
R_{n}(X)=\mathbb{I}_{n} \operatorname{Tr} X-X,
$$

for $X \in \mathcal{B}\left(\mathbb{C}^{n}\right)$. It is well known that $R_{n}$ is completely co-positive (i.e. $R_{n} \circ T$ is $\mathrm{CP}$ ) and hence optimal. Recently, this map was generalized by Breuer and Hall [36, 37] to the following family of positive maps

$$
\Phi_{2 k}^{U}(X)=\frac{1}{2(k-1)}\left(R_{2 k}(X)-U X^{T} U^{\dagger}\right),
$$

where $U$ is an arbitrary antisymmetric unitary $2 k \times 2 k$ matrix. It was shown that these map are indecomposable [36, 37] and optimal [36]. Such antisymmetric unitary matrix may be easily construct as follows

$$
U=V U_{0} V^{\dagger},
$$

where $V$ stands for real orthogonal matrix $\left(V V^{\dagger}=\right.$ $\left.V V^{T}=\mathbb{I}_{2 k}\right)$ and

$$
U_{0}=\mathbb{I}_{k} \otimes J,
$$

with $J$ being $2 \times 2$ symplectic matrix

$$
J=\left(\begin{array}{cc}
0 & 1 \\
-1 & 0
\end{array}\right)
$$

It is therefore clear that in this case one has

$$
\Phi_{2 k}^{U}(X)=V \Phi_{2 k}^{0}\left(V^{\dagger} X V\right) V^{\dagger},
$$

where $\Phi_{2 k}^{0}$ corresponds to $\Phi_{2 k}^{U}$ with $U=U_{0}$. Actually, one can always find a basis in $\mathbb{C}^{2 k}$ such that $U$ takes the "canonical form" $U_{0}$. Interestingly for $k=2$ the Breuer-Hall map $\Phi_{4}^{0}$ reproduces well known Robertson map 28] who provided it as an example of an extremal (and hence optimal) indecomposable positive map. Moreover, Robertson construction may be nicely described in terms of $R_{2}$ as follows 39]

$$
\Phi_{4}^{0}\left(\begin{array}{c|c}
X_{11} & X_{12} \\
\hline X_{21} & X_{22}
\end{array}\right)=\frac{1}{2}\left(\begin{array}{c|c}
\mathbb{I}_{2} \operatorname{Tr} X_{22} & -\left[X_{12}+R_{2}\left(X_{21}\right)\right] \\
\hline-\left[X_{21}+R_{2}\left(X_{12}\right)\right] & \mathbb{I}_{2} \operatorname{Tr} X_{11}
\end{array}\right),
$$

where $X_{k l} \in \mathcal{B}\left(\mathbb{C}^{2}\right)$. This pattern is reproduced for may be represented as follows: arbitrary $k$. It is easy to show that the action of $\Phi_{2 k}^{0}$

$\Phi_{2 k}^{0}\left(\begin{array}{c|c|c|c}X_{11} & X_{12} & \cdots & X_{1 k} \\ \hline X_{21} & X_{22} & \cdots & X_{2 k} \\ \hline \vdots & \vdots & \ddots & \vdots \\ \hline X_{k 1} & X_{k 2} & \cdots & X_{k k}\end{array}\right)=\frac{1}{2(k-1)}\left(\begin{array}{c|c|c|c}\mathbb{I}_{2}\left(\operatorname{Tr} X-\operatorname{Tr} X_{11}\right) & -\left(X_{12}+R_{2}\left(X_{21}\right)\right) & \cdots & -\left(X_{1 k}+R_{2}\left(X_{k 1}\right)\right) \\ \hline-\left(X_{21}+R_{2}\left(X_{12}\right)\right) & \mathbb{I}_{2}\left(\operatorname{Tr} X-\operatorname{Tr} X_{22}\right) & \cdots & -\left(X_{2 k}+R_{2}\left(X_{k 2}\right)\right) \\ \hline \vdots & \vdots & \ddots & \vdots \\ \hline-\left(X_{k 1}+R_{2}\left(X_{1 k}\right)\right) & -\left(X_{k 2}+R_{2}\left(X_{2 k}\right)\right) & \cdots & \mathbb{I}_{2}\left(\operatorname{Tr} X-\operatorname{Tr} X_{k k}\right)\end{array}\right)$,

where again $X_{i j}$ are $2 \times 2$ blocks. Hence $\Phi_{2 k}^{0}$ is defined in (15) by $R_{2 k}$ but the above pattern shows that it basically uses reduction map $R_{2}$ only. We stress that $R_{2}$ is exceptional: it is not only optimal but also ex- 
tremal. Indeed, the corresponding entanglement witness $W_{2}=2\left(1 \otimes R_{2}\right) P_{2}^{+}$reads as follows

$$
W_{2}=\mathbb{I}_{2} \otimes \mathbb{I}_{2}-P_{2}^{+}=\left(\begin{array}{cc|cc}
\cdot & \cdot & \cdot & -1 \\
\cdot & 1 & \cdot & \cdot \\
\hline \cdot & \cdot & 1 & \cdot \\
-1 & \cdot & \cdot & \cdot
\end{array}\right) .
$$

and $W_{2}=P^{\Gamma}$, where $P=|\psi\rangle\langle\psi|$ with

$$
|\psi\rangle=|01\rangle-|10\rangle \text {. }
$$

Note, that $R_{2}$ may be nicely represented as follows

$$
R_{2}(X)=J X^{T} J^{\dagger}
$$

with $J$ defined in (18). Formula (23) provides Kraus representation for $R_{2} \circ T$ and shows that $R_{2}$ is completely co-positive.
In the present paper we propose another construction of maps in $\mathcal{B}\left(\mathbb{C}^{2 k}\right)$. Now, instead of treating a $2 k \times 2 k$ matrix $X$ as a $k \times k$ matrix with $2 \times 2$ blocks $X_{i j}$ we consider alternative possibility, i.e. we consider $X$ as a $2 \times 2$ with $k \times k$ blocks and define

$$
\Psi_{2 k}^{0}\left(\begin{array}{c|c}
X_{11} & X_{12} \\
\hline X_{21} & X_{22}
\end{array}\right)=\frac{1}{k}\left(\begin{array}{c|c}
\mathbb{I}_{k} \operatorname{Tr} X_{22} & -\left[X_{12}+R_{k}\left(X_{21}\right)\right] \\
\hline-\left[X_{21}+R_{k}\left(X_{12}\right)\right] & \mathbb{I}_{k} \operatorname{Tr} X_{11}
\end{array}\right)
$$

Again, normalization factor guaranties that the map is unital, i.e. $\Psi_{2 k}^{0}\left(\mathbb{I}_{2} \otimes \mathbb{I}_{k}\right)=\mathbb{I}_{2} \otimes \mathbb{I}_{k}$. It is clear that for $k=2$ one has

$$
\Phi_{4}^{0}=\Psi_{4}^{0} .
$$

We stress that our new construction is much simpler than $\Phi_{2 k}^{0}$ and it uses as a building block the true reduction map in $\mathcal{B}\left(\mathbb{C}^{k}\right)$. Moreover, it is clear that it provides a natural generalization of the original Robertson map in $\mathcal{B}\left(\mathbb{C}^{4}\right)$.

Now, our task is to prove that $\Psi_{2 k}^{0}$ defines a positive map. It is enough to show that each rank-1 projector $P$ is mapped via $\Psi_{2 k}^{0}$ into a positive element in $\mathcal{B}\left(\mathbb{C}^{2 k}\right)$, that is, $\Psi_{2 k}^{0}(P) \geq 0$. Let $P=|\psi\rangle\langle\psi|$ with arbitrary $\psi$ from $\mathbb{C}^{2 k}$. Now, due to $\mathbb{C}^{2 k}=\mathbb{C}^{k} \oplus \mathbb{C}^{k}$ one has

$$
\psi=\psi_{1} \oplus \psi_{2},
$$

with $\psi_{1}, \psi_{2} \in \mathbb{C}^{k}$ and hence

$$
P=\left(\begin{array}{c|c}
X_{11} & X_{12} \\
\hline X_{21} & X_{22}
\end{array}\right)=\left(\begin{array}{c|c}
\left|\psi_{1}\right\rangle\left\langle\psi_{1}\right| & \left|\psi_{1}\right\rangle\left\langle\psi_{2}\right| \\
\hline\left|\psi_{2}\right\rangle\left\langle\psi_{1}\right| & \left|\psi_{2}\right\rangle\left\langle\psi_{2}\right|
\end{array}\right) .
$$

One has therefore

$$
\Psi_{2 k}^{0}(P)=\frac{1}{k}\left(\begin{array}{c|c}
\mathbb{I}_{k} \operatorname{Tr} X_{22} & -A \\
\hline-A^{\dagger} & \mathbb{I}_{k} \operatorname{Tr} X_{11}
\end{array}\right),
$$

where the linear operator $A: \mathbb{C}^{k} \rightarrow \mathbb{C}^{k}$ reads as follows

$$
A=\left|\psi_{1}\right\rangle\left\langle\psi_{2}|-| \psi_{2}\right\rangle\left\langle\psi_{1}\right|+\left\langle\psi_{1} \mid \psi_{2}\right\rangle \mathbb{I}_{k} .
$$

Let $\left\langle\psi_{j} \mid \psi_{j}\right\rangle=a_{j}^{2}>0$ (if one of $a_{j}$ vanishes then evidently one has $\left.\Psi_{2 k}^{0}(P) \geq 0\right)$. Defining

$$
L=\sqrt{k}\left(\begin{array}{c|c}
\mathbb{I}_{k} a_{2}^{-1} & \mathbb{O}_{k} \\
\hline \mathbb{O}_{k} & \mathbb{I}_{k} a_{1}^{-1}
\end{array}\right),
$$

one finds

$$
L \Psi_{2 k}^{0}(P) L^{\dagger}=\left(\begin{array}{c|c}
\mathbb{I}_{k} & -\widetilde{A} \\
\hline-\widetilde{A}^{\dagger} & \mathbb{I}_{k}
\end{array}\right)
$$

with

$$
\widetilde{A}=\left|\widetilde{\psi}_{1}\right\rangle\left\langle\widetilde{\psi}_{2}|-| \widetilde{\psi}_{2}\right\rangle\left\langle\widetilde{\psi}_{1}\right|+\left\langle\widetilde{\psi}_{1} \mid \widetilde{\psi}_{2}\right\rangle \mathbb{I}_{k},
$$

and normalized $\widetilde{\psi}_{j}=\psi_{j} / a_{j}$. Hence, to show that $\Psi_{2 k}^{0}(P) \geq 0$ one needs to prove

$$
\left(\begin{array}{c|c}
\mathbb{I}_{k} & -\widetilde{A} \\
\hline-\widetilde{A}^{\dagger} & \mathbb{I}_{k}
\end{array}\right) \geq 0
$$

for arbitrary $\psi_{j} \neq 0$. Now, the above condition is equivalent to

$$
\widetilde{A} \widetilde{A}^{\dagger} \leq \mathbb{I}_{k} .
$$

Vectors $\left\{\psi_{1}, \psi_{2}\right\}$ span 2-dimensional subspace in $\mathbb{C}^{k}$ and let $\left\{e_{1}, e_{2}\right\}$ be a 2-dim. orthonormal basis such that $\psi_{1}=e_{1}$ and

$$
\psi_{2}=e^{i \lambda} s e_{1}+c e_{2},
$$


with $s=\sin \alpha, c=\cos \alpha$ for some angle $\alpha$. Now, completing the basis $\left\{e_{1}, e_{2}, e_{3}, \ldots, e_{k}\right\}$ in $\mathbb{C}^{k}$ one easily finds that the matrix elements of $\widetilde{A}$ has a form of the following direct sum

$$
\widetilde{A}=\left(\begin{array}{c|c}
e^{-i \lambda} s & c \\
\hline-c & e^{i \lambda} s
\end{array}\right) \oplus e^{-i \lambda} s \mathbb{I}_{k-2} .
$$

Hence

$$
\widetilde{A} \widetilde{A}^{\dagger}=\mathbb{I}_{2} \oplus s^{2} \mathbb{I}_{k-2}
$$

which proves (34) since all eigenvalues of $\widetilde{A} \widetilde{A}^{\dagger}-$ $\left\{1,1, s^{2}, \ldots, s^{2}\right\}$ - are bounded by 1 .

Now, our new positive maps can be useful in detecting entanglement only if they are not completely positive. It is easy to check that the corresponding Choi matrix

$$
W_{2 k}=\sum_{i, j=1}^{2 k} e_{i j} \otimes \Psi_{2 k}^{0}\left(e_{i j}\right)
$$

possesses 2 negative eigenvalues $\{-1,(2-k) / k\}$ (unless $k=2$ ). Hence, (38) defines true entanglement witness in $\mathbb{C}^{2 k} \otimes \mathbb{C}^{2 k}$. As usual using Dirac notation we define $e_{k l}:=\left|e_{k}\right\rangle\left\langle e_{l}\right|$. Note, that the corresponding Brauer-Hall witness possesses only one negative eigenvalue " -1 ". Hence these two classes are different (unless $k=2$ ).

\section{PROPERTIES OF NEW ENTANGLEMENT WITNESSES}

In this section we study basis properties of $W_{2 k}$.

\section{A. $W_{2 k}$ are indecomposable}

To show that $W_{2 k}$ is indecomposable one needs to define a PPT state $\rho$ in $\mathbb{C}^{2 k} \otimes \mathbb{C}^{2 k}$ such that
$\operatorname{Tr}\left(W_{2 k} \rho_{2 k}\right)<0$. Consider the following operator

$$
\rho_{2 k}=\sum_{i, j=1}^{2 k} e_{i j} \otimes \rho_{i j}^{(2 k)}
$$

where the $2 k \times 2 k$ blocks are defined as follows: diagonal blocks

$$
\rho_{i i}^{(2 k)}=N_{k}\left(\begin{array}{c|c}
k \mathbb{I}_{k} & \mathbb{O}_{k} \\
\hline \mathbb{O}_{k} & \mathbb{I}_{k}
\end{array}\right),
$$

for $i=1, \ldots, k$, and

$$
\rho_{i i}^{(2 k)}=N_{k}\left(\begin{array}{c|c}
\mathbb{I}_{k} & \mathbb{O}_{k} \\
\hline \mathbb{O}_{k} & k \mathbb{I}_{k}
\end{array}\right),
$$

for $i=k+1, \ldots, 2 k$. The off-diagonal blocks are form:

$$
\rho_{i, i+k}^{(2 k)}=-N_{k} W_{i, i+k}^{(2 k)}
$$

for $i=1, \ldots, k$,

$$
\rho_{i j}^{(2 k)}=N_{k} e_{i j}
$$

for $i=1, \ldots, k, j=k+1, \ldots, 2 k$ and $j \neq i+k$ and

$$
\rho_{i j}^{(2 k)}=\mathbb{O}_{k}
$$

otherwise. The normalization factor $N_{k}$ is given by

$$
1 / N_{k}=2 k^{2}(k+1)
$$

Direct calculation shows that

$$
\rho \geq 0, \quad \rho^{\Gamma} \geq 0, \quad \operatorname{Tr} \rho=1 .
$$

For example for $k=2$ one obtains the following density operator 


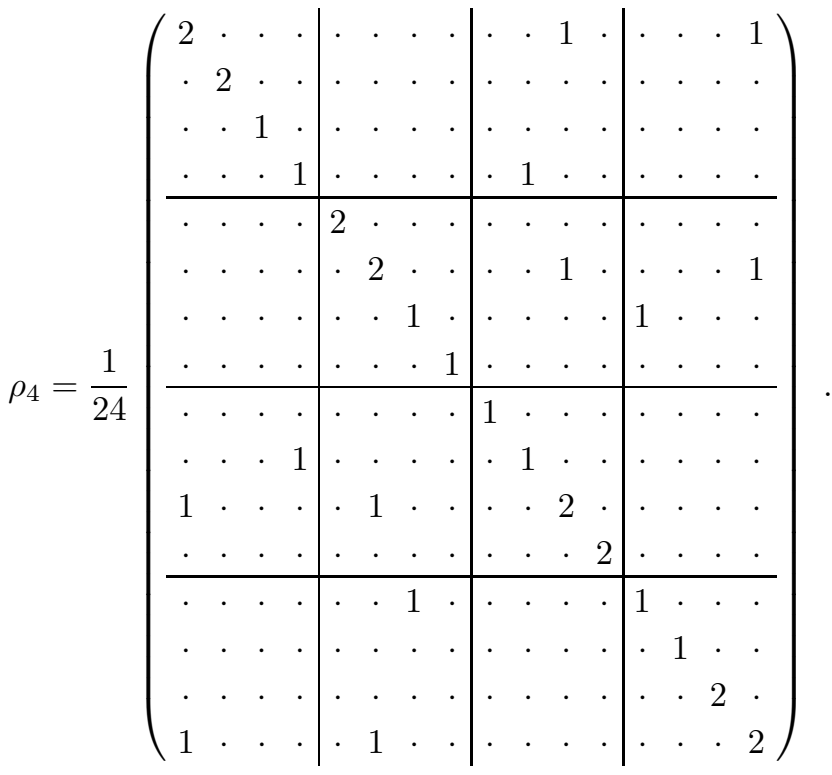

One easily finds for the trace

$$
\operatorname{Tr}\left(W_{2 k} \rho_{2 k}\right)=-\frac{k-1}{k^{2}(k+1)},
$$

which proves indecomposability of $W_{2 k}$.

\section{B. $W_{2 k}$ are atomic}

In order to prove that $W_{2 k}$ is atomic one has to define a PPT state $D_{2 k}$ such that Schmidt rank of $D_{2 k}$ and of its partial transposition $D_{2 k}^{\Gamma}$ is bounded by 2 and show that $\operatorname{Tr}\left(W_{2 k} D_{2 k}\right)<0$. It is clear that atomicity implies indecomposability but for clarity of presentation we treat these two notions independently. Let us introduce the following family of product vectors

$$
\begin{aligned}
\phi_{1} & =e_{1} \otimes e_{1}, \\
\phi_{2} & =e_{1} \otimes e_{k+1}, \\
\phi_{3} & =e_{k} \otimes e_{1}, \\
\phi_{4} & =e_{k} \otimes e_{2 k} \\
\phi_{5} & =e_{k+1} \otimes e_{1}, \\
\phi_{6} & =e_{k+1} \otimes e_{k+1}, \\
\phi_{7} & =e_{k+1} \otimes e_{2 k} .
\end{aligned}
$$

Define now the following positive operator

$$
\begin{aligned}
D_{2 k} & =\frac{1}{7}\left(\left|\phi_{1}+\phi_{6}\right\rangle\left\langle\phi_{1}+\phi_{6}|+| \phi_{5}-\phi_{4}\right\rangle\left\langle\phi_{5}-\phi_{4}\right|\right. \\
& \left.+\left|\phi_{2}\right\rangle\left\langle\phi_{2}|+| \phi_{3}\right\rangle\left\langle\phi_{3}|+| \phi_{7}\right\rangle\left\langle\phi_{7}\right|\right)
\end{aligned}
$$

One easily finds for its partial transposition

$$
\begin{aligned}
D_{2 k}^{\Gamma} & =\frac{1}{7}\left(\left|\phi_{2}+\phi_{5}\right\rangle\left\langle\phi_{2}+\phi_{5}|+| \phi_{3}-\phi_{7}\right\rangle\left\langle\phi_{3}-\phi_{7}\right|\right. \\
& \left.+\left|\phi_{1}\right\rangle\left\langle\phi_{1}|+| \phi_{4}\right\rangle\left\langle\phi_{4}|+| \phi_{6}\right\rangle\left\langle\phi_{6}\right|\right)
\end{aligned}
$$

Now, it is clear from that both $D_{2 k}$ and $D_{2 k}^{\Gamma}$ are constructed out of rank-1 projectors and Schmidt rank of each projector is 1 or 2 . Therefore

$$
\mathrm{SN}\left(D_{2 k}\right) \leq 2, \quad \mathrm{SN}\left(D_{2 k}^{\Gamma}\right) \leq 2 .
$$

Finally, one finds for the trace

$$
\operatorname{Tr}\left(W_{2 k} D_{2 k}\right)=-\frac{1}{7 k}
$$

which shows that $W_{2 k}$ defines atomic entanglement witness.

\section{C. $W_{2 k}$ are optimal}

To show that $W_{2 k}$ is optimal we use the following result Lewenstein et. al. [7]: if the family of product vectors $\psi \otimes \phi \in \mathbb{C}^{2 k} \otimes \mathbb{C}^{2 k}$ satisfying

$$
\langle\psi \otimes \phi|W| \psi \otimes \phi\rangle=0
$$

span the total Hilbert space $\mathbb{C}^{2 k} \otimes \mathbb{C}^{2 k}$, then $W$ is optimal. Let us introduce the following sets of vectors:

$$
f_{m n}=e_{m}+e_{n},
$$


and

$$
g_{m n}=e_{m}+i e_{n},
$$

for each $1 \leq m<n \leq 2 k$. It is easy to check that $(2 k)^{2}$ vectors $\psi_{\alpha} \otimes \psi_{\alpha}^{*}$ with $\psi_{\alpha}$ belonging to the set

$$
\left\{e_{l}, f_{m n}, g_{m n}\right\},
$$

are linearly independent and hence they do span $\mathbb{C}^{2 k} \otimes \mathbb{C}^{2 k}$. Direct calculation shows that

$$
\left\langle\psi_{\alpha} \otimes \psi_{\alpha}^{*}|W| \psi_{\alpha} \otimes \psi_{\alpha}^{*}\right\rangle=0,
$$

which proves that $W_{2 k}$ is an optimal EW.

\section{D. $W_{2 k}$ have circulant structure}

Finally, let us note that $W_{2 k}$ displays so called circulant structure [47, 48]. Let $\left\{e_{1}, \ldots, e_{d}\right\}$ be an orthonormal basis in $\mathbb{C}^{d}$ and let $S: \mathbb{C}^{d} \rightarrow \mathbb{C}^{d}$ be a shift operator (elementary permutation) defined by

$$
S e_{j}=e_{j+1}, \quad j=1, \ldots, d \quad(\bmod d) .
$$

Now, introducing

$$
\Sigma_{0}=\operatorname{span}\left\{e_{1} \otimes e_{1}, \ldots, e_{d} \otimes e_{d}\right\},
$$

define

$$
\Sigma_{\alpha}=\left(\mathbb{1} \otimes S^{\alpha}\right) \Sigma_{1}, \quad \alpha=0, \ldots, d-1 .
$$

A bipartite operator $X: \mathbb{C}^{d} \otimes \mathbb{C}^{d} \rightarrow \mathbb{C}^{d} \otimes \mathbb{C}^{d}$ displays circulant structure if

$$
X=X_{0} \oplus \ldots \oplus X_{d-1},
$$

such that each $X_{\alpha}$ is supported on $\Sigma_{\alpha}$. It is therefore clear that

$$
X_{\alpha}=\sum_{i, j=1}^{d} x_{i j}^{(\alpha)} e_{i j} \otimes S^{\alpha} e_{i j} S^{\alpha \dagger},
$$

where $\left[x_{i j}^{(\alpha)}\right]$ is $d \otimes d$ complex matrix for each $\alpha=$ $0, \ldots, d-1$, i.e. a circulant bipartite operator $X$ is uniquely defined by the collection of $d$ complex matrices $\left[x_{i j}^{(\alpha)}\right]$.

\section{STRUCTURAL PHYSICAL APPROXIMATION}

It is well know that positive maps cannot be directly implemented in the laboratory. The idea of structural physical approximation (SPA) [42, 43] is to mix a positive map $\Lambda$ with some completely positive map making the mixture $\widetilde{\Lambda}$ completely positive. In the recent paper [44] the authors analyze SPA to a positive map $\Lambda: \mathcal{H}_{\mathcal{A}} \rightarrow \mathcal{H}_{\mathcal{B}}$ obtained through minimal admixing of white noise

$$
\widetilde{\Lambda}(\rho)=p \frac{\mathbb{I}_{B}}{d_{B}} \operatorname{Tr}(\rho)+(1-p) \Lambda(\rho) .
$$

The minimal means that the positive mixing parameter $0<p<1$ is the smallest one for which the resulting map $\widetilde{\Lambda}$ is completely positive, i.e. it defines a quantum channel. Equivalently, one may introduce SPA of an entanglement witness $W$ :

$$
\widetilde{W}=\frac{p}{d_{A} d_{B}} \mathbb{I}_{A} \otimes \mathbb{I}_{B}+(1-p) W,
$$

where $p$ is the smallest parameter for which $\widetilde{W}$ is a positive operator in $\mathcal{H}_{A} \otimes \mathcal{H}_{B}$, i.e. it defines (possibly unnormalized) state.

It was conjectured 44 that SPA to optimal positive maps correspond to entanglement breaking maps (channels). Equivalently, SPA to optimal entanglement witnesses correspond to separable (unnormalized) states. It turns out that the family of optimal maps/witnesses constructed in this paper does support this conjecture.

The corresponding SPA of $W_{2 k}$ is given by

$$
\widetilde{W}_{2 k}=\frac{p}{(2 k)^{2}} \mathbb{I}_{2 k} \otimes \mathbb{I}_{2 k}+(1-p) W_{2 k} .
$$

Using the fact that the maximal negative eigenvalue of $W_{2 k}$ equals " -1 " one easily finds the following condition for the positivity of $\widetilde{W}_{2 k}$

$$
p \geq \frac{d}{d+1},
$$

with $d=2 k$. Surprisingly, one obtains the same bound for $p$ as in Eqs. (26), (33) and (65) in [44].

Now, to show that SPA of $\Psi_{2 k}^{0}$ (or eqivalently $W_{2 k}$ ) is entanglement breaking (equivalently separable) we use the following

Lemma 1 [49] Let $\Lambda: \mathcal{B}\left(\mathbb{C}^{d}\right) \rightarrow \mathcal{B}\left(\mathbb{C}^{d}\right)$ be a positive unital map. Then $S P A$ of $\Lambda$ is entanglement breaking if $\Lambda$ detects all entangled isotropic states in $\mathbb{C}^{d} \otimes \mathbb{C}^{d}$.

Indeed, let

$$
\rho_{p}=\frac{p}{d^{2}} \mathbb{I}_{d} \otimes \mathbb{I}_{d}+(1-p) P_{d}^{+},
$$

be an isotropic state which is known to be entangled iff

$$
p<\frac{1}{d+1}
$$


Now, assume that $(\mathbb{1} \otimes \Lambda) \rho_{p}$ is not positive if $\rho_{p}$ is entangled. Using $\Lambda\left(\mathbb{I}_{d}\right)=\mathbb{I}_{d}$ one obtains

$$
(\mathbb{1} \otimes \Lambda) \rho_{p}=\frac{p}{d^{2}} \mathbb{I}_{d} \otimes \mathbb{I}_{d}+(1-p) W,
$$

that is, $(\mathbb{1} \otimes \Lambda) \rho_{p}=\widetilde{W}$. Now, if $\widetilde{W}$ is positive, then $\rho_{p}$ has to be separable (otherwise it would be detected by $\Lambda$ ). But since $\mathbb{1} \otimes \Lambda$ sends separable states into separable states one concludes that $\widetilde{W}$ is separable (or equivalently $\widetilde{\Lambda}$ is entanglement breaking).

Lemma 2 If in addition $\Lambda$ is self-dual, i.e.

$$
\operatorname{Tr}(A \cdot \Lambda(B))=\operatorname{Tr}(\Lambda(A) \cdot B),
$$

for all $A, B \in \mathcal{B}\left(\mathbb{C}^{d}\right)$, then it is enough to check whether all entangled isotropic states are detected by the witness $W$, i.e. $\operatorname{Tr}\left(W \rho_{p}\right)<0$ for all $p$ satisfying (62).

Again, the proof is very easy. One has

$$
\begin{aligned}
\operatorname{Tr}\left(\rho_{p} \cdot W\right) & =\operatorname{Tr}\left(\rho_{p} \cdot(\mathbb{1} \otimes \Lambda) P_{d}^{+}\right) \\
& =\operatorname{Tr}\left((\mathbb{1} \otimes \Lambda) \rho_{p} \cdot P_{d}^{+}\right),
\end{aligned}
$$

where in the last equality we used the self-duality of $\Lambda$. Now, if $\operatorname{Tr}\left(\rho_{p} \cdot W\right)<0$ for $p$ satisfying (62), then $(\mathbb{1} \otimes \Lambda) \rho_{p}$ is not positive (otherwise its trace with the projector $P_{d}^{+}$would be positive). Hence by Lemma 1 SPA $\widetilde{\Lambda}$ is entanglement breaking.

We are prepared to show that SPA for $\Psi_{2 k}^{0}$ is entanglement breaking.

Lemma $3 \Psi_{2 k}^{0}$ is self-dual.

One checks by direct calculations that

$$
\operatorname{Tr}\left(e_{k l} \cdot \Psi_{2 k}^{0}\left(e_{m n}\right)\right)=\operatorname{Tr}\left(\Psi_{2 k}^{0}\left(e_{k l}\right) \cdot e_{m n}\right),
$$

for all $k, l, m, n=1, \ldots, 2 k$. Hence, due to the Lemma 2 , to show that SPA for $\Psi_{2 k}^{0}$ is entanglement breaking is it enough to prove

Lemma $4 \operatorname{Tr}\left(W_{2 k} \rho_{p}\right)<0$ for all $p$ satisfying (62).

To prove it let us note that

$$
\operatorname{Tr}\left(W_{2 k} \rho_{p}\right)=\frac{p}{(2 k)^{2}} \operatorname{Tr} W_{2 k}+(1-p) \operatorname{Tr}\left(W_{2 k} P_{2 k}^{+}\right) .
$$

Now, $\operatorname{Tr} W_{2 k}=2 k$, and

$$
\operatorname{Tr}\left(W_{2 k} P_{2 k}^{+}\right)=\frac{1}{2 k} \sum_{m, n=1}^{2 k}\left\langle m\left|\Psi_{2 k}^{0}\left(e_{m n}\right)\right| n\right\rangle .
$$

Finally, using definition of $\Psi_{2 k}^{0}$ one gets

$$
\sum_{m, n=1}^{2 k}\left\langle m\left|\Psi_{2 k}^{0}\left(e_{m n}\right)\right| n\right\rangle=-2 k,
$$

and hence

$$
\operatorname{Tr}\left(W_{2 k} \rho_{p}\right)=\frac{p(d+1)-1}{d},
$$

with $d=2 k$. Now, if $\rho_{p}$ is entangled, i.e. $p<1 /(d+1)$, then $\operatorname{Tr}\left(W_{2 k} \rho_{p}\right)<0$ which shows that $W_{2 k}$ detects all entangled isotropic states.

\section{CONCLUSIONS}

We have provided a new construction of EWs in $\mathbb{C}^{d} \otimes \mathbb{C}^{d}$ with $d=2 k$. It was shown that these EWs are indecomposable, i.e. they are able to detect PPT entangled state. Moreover, they are so called atomic EWs, i.e. they able to detect PPT entangled states $\rho$ such that both $\rho$ and $\rho^{\Gamma}$ possess Schmidt number 2 . The crucial property of our witnesses is they optimality, i.e. they are no other witnesses which can detect more entangled states.

Equivalently, our construction gives rise to the new class of positive maps in algebras of $d \times d$ complex matrices. For $k=2$ this construction reproduces old example of Robertson map [28] and hence [39] defines the special case of Brauer-Hall maps [36, 37].

Let us observe that if $\Lambda: \mathcal{B}\left(\mathbb{C}^{d}\right) \rightarrow \mathcal{B}\left(\mathbb{C}^{d}\right)$ is a positive indecomposable map then for any unitaries $U_{1}, U_{2}: \mathbb{C}^{d} \rightarrow \mathbb{C}^{d}$ a new map

$$
\Lambda^{U_{1} U_{2}}(A):=U_{1} \Lambda\left(U_{2}^{\dagger} A U_{2}\right) U_{1}^{\dagger},
$$

is again positive and indecomposable [39]. This observation enables one to generalize Robertson map $\Phi_{2 k}^{0}$ and our new map $\Psi_{2 k}^{0}$ to $\Phi_{2 k}^{U_{1} U_{2}}$ and $\Psi_{2 k}^{U_{1} U_{2}}$. Note, that if $U_{1}=U_{2}=U$ given by (15), then $\Phi_{2 k}^{U}:=\Phi_{2 k}^{U U}$ defines Breuer-Hall map. Therefore, $\Psi_{2 k}^{U}:=\Psi_{2 k}^{U U}$ may be regarded as a Breuer-Hall-like generalization of our primary map $\Psi_{2 k}^{0}$.

It should be stressed, an EW defined by the BreuerHall map and EW $W_{2 k}$ introduces in this paper are different, i.e. they do detect different classes of PPT entangled states. Direct calculation shows that the PPT entangled state (39) is not detected by the Breuer-Hall witness. On the other hand consider the family of PPT entangled state introduced in 36]

$$
\rho(\lambda)=\lambda P_{d}^{+}+(1-\lambda) \rho_{0},
$$

with

$$
\rho_{0}=\frac{2}{d(d+1)} U_{0} P_{S} U_{0}^{\dagger}
$$

and $P_{S}$ being the projector onto the subspace of states symmetric under the swap operation. It was shown that $\rho(\lambda)$ is PPT for $0 \leq \lambda \leq 1(d+2)$. Moreover, 
Breuer-Hall witness detects all entangled states within $\lambda$-family (69) (both PPT and NPT). Direct calculation shows that our witness $W_{2 k}$ does not detect PPT entangled states in (69).

Interestingly, the partial transposition $W_{2 k}^{\Gamma}$ defines an EW with $k(k-1) / 2$ negative eigenvalues (all equal to ' $\left.-1^{\prime}\right)$. For $k=2$ it gives exactly one negative eigenvalue (the fact well known from the family of Breuer-Hall maps in 4 dimensions). Therefore, this example provide an EW with multiple negative eigenvalues. However, contrary to the Breuer-Hall maps we were not able to show that $W_{2 k}^{\Gamma}$ is optimal.

We have shown that structural physical approximation for our new class of positive maps gives rise to entanglement breaking channels. This result supports recent conjecture by Korbicz et. al. 44].

Finally, let us mention some open questions. In this paper we have used reduction map as a building block to construct other optimal maps. Can we use other positive maps as building blocks? Is it true that properties of building blocks (like optimality and/or atomicity) are shared by the map which is built out of them?

\section{Acknowledgement}

We thank Antonio Acin and Andrzej Kossakowski for valuable discussions. This work was partially supported by the Polish Ministry of Science and Higher Education Grant No 3004/B/H03/2007/33 and by the Polish Research Network Laboratory of Physical Foundations of Information Processing.
[1] M. A. Nielsen and I. L. Chuang, Quantum Computation and Quantum Information, (Cambridge University Press, Cambridge, England, 2000).

[2] R. Horodecki, P. Horodecki, M. Horodecki and K. Horodecki, Rev. Mod. Phys. 81, 865 (2009).

[3] M. Horodecki, P. Horodecki and R. Horodecki, Phys. Lett. A 223, 1 (1996).

[4] B.M. Terhal, Phys. Lett. A 271, 319 (2000).

[5] B. M. Terhal, Theor. Comput. Sci. 287, 313 (2002).

[6] M. Lewenstein, B. Kraus, J. I. Cirac, and P. Horodecki, Phys. Rev. A 62, 052310 (2000).

[7] M. Lewenstein, B. Kraus, P. Horodecki, and J. I. Cirac, Phys. Rev. A 63, 044304 (2001).

[8] B. Kraus, M. Lewenstein, and J. I. Cirac, Phys. Rev. A 65, 042327 (2002).

[9] P. Hyllus, O. Gühne, D. Bruss, and M. Lewenstein Phys. Rev. A 72, 012321 (2005).

[10] D. Bruß, J. Math. Phys. 43, 4237 (2002).

[11] G. Tóth and O. Gühne, Phys. Rev. Lett. 94, 060501 (2005)

[12] R. A. Bertlmann, H. Narnhofer and W. Thirring, Phys. Rev. A 66, 032319 (2002).

[13] F.G.S.L. Brandão, Phys. Rev. A 72, 022310 (2005).

[14] G. Sarbicki, J. Phys. A: Math. Theor. 41, 375303 (2008).

[15] D. Chruściński and A. Kossakowski, J. Phys. A: Math. Theor. 41, 145301 (2008).

[16] M. Bourennane, M. Eibl, C. Kurtsiefer, S. Gaertner, H. Weinfurter, O. Gühne, P. Hyllus, D. Bruss, M. Lewenstein and A. Sanpera, Phys. Rev. Lett. 92, 087902 (2004).

[17] L.-A. Wu, S. Bandyopadhyay, M. S. Sarandy, and D. A. Lidar, Phys. Rev. A 72, 032309 (2005).

[18] A.C. Doherty, P.A. Parrilo and F.M. Spedalieri, Phys. Rev. Lett. 88, 187904 (2002).

[19] F.G.S.L. Brandão and R.O. Vianna, Phys. Rev. Lett. 93, 220503 (2004).

[20] J. Eisert, P. Hyllus, O. Gühne, and M. Curty, Phys.
Rev. A 70, 062317 (2004);

[21] P. Hyllus, O. Guehne, D. Bruß, M. Lewenstein, Phys. Rev. A 72, 012321 (2005).

[22] M.-D. Choi, Lin. Alg. Appl. 10, 285 (1975).

[23] A. Jamiołkowski, Rep. Math. Phys. 3, 275 (1972).

[24] S.L. Woronowicz, Rep. Math. Phys. 10, 165 (1976).

[25] E. Størmer, Acta Math. 110, 233 (1963).

[26] M.-D. Choi, Lin. Alg. Appl. 12, 95 (1975); M.-D. Choi, J. Operator Theory, 4, 271 (1980).

[27] S.L. Woronowicz, Comm. Math. Phys. 51, 243 (1976).

[28] A.G. Robertson, Math. Proc. Camb. Phil. Soc., 94, 71 (1983). 34, 87 (1983); A.G. Robertson, J. London Math. Soc. (2) 32, 133 (1985).

[29] W.-S. Tang, Lin. Alg. Appl. 79, 33 (1986).

[30] K. Tanahashi and J. Tomiyama, Canad. Math. Bull. 31, 308 (1988).

[31] H. Osaka, Lin. Alg. Appl. 153, 73 (1991); ibid. 186, 45 (1993).

[32] F. Benatti, R. Floreanini and M. Piani, Phys. Lett. A 326, 187 (2004).

[33] K.-C. Ha, Publ. RIMS, Kyoto Univ. 34, 591 (1998)

[34] K.-C. Ha and S.-H. Kye, J. Phys. A: Math. Gen. 38, 9039 (2005); Phys. Lett. A 325, 315 (2004).

[35] A. Kossakowski, Open Sys. Information Dyn. 10, 213 (2003).

[36] H.-P. Breuer, Phys. Rev. Lett. 97, 0805001 (2006).

[37] W. Hall, J. Phys. A: Math. Gen. 39, (2006) 14119.

[38] D. Chruściński and A. Kossakowski, Open Systems and Inf. Dynamics, 14, 275 (2007).

[39] D. Chruściński and A. Kossakowski, J. Phys. A: Math. Theor. 41, 215201 (2008).

[40] D. Chruściński and A. Kossakowski, Spectral conditions for positive maps, arXiv:0809.4909 (to be published in Comm. Math. Phys.)

[41] D. Chruściński and A. Kossakowski, Phys. Lett. A 373, 2301 (2009).

[42] P. Horodecki, Phys. Rev. A 68, 052101 (2003).

[43] P. Horodecki and A. Ekkert, Phys. Rev. Lett. 89, 
127902 (2002).

[44] J.K. Korbicz, M.L. Almeida, J. Bae, M. Lewenstein and A. Acin, Phys. Rev. A 78, 062105 (2008).

[45] B. Terhal and P. Horodecki, Phys. Rev. A 61, 040301 (2000); A. Sanpera, D. Bruss and M. Lewenstein, Phys. Rev. A 63, 050301(R) (2001)

[46] M. Horodecki and P. Horodecki, Phys. Rev. A 59, 4206 (1999).
[47] D. Chruściński and A Kossakowski, Phys. Rev. A 74, 022308 (2006)

[48] D. Chruściński and A Kossakowski, Phys. Rev. A 76, 032308 (2007); D. Chruściński and A. Pittenger, J. Phys. A: Math. Theor. 41, 385301 (2008).

[49] We thank Antonio Acin for his remarks. 